6. Now, let us consider $s$ as a complex variable and $x$ as a function of $s$. Then equation (10) can easily be solved; we obtain

$$
x=\alpha e^{-s^{2} / 4 \omega}+\frac{1}{2 \omega} \int_{0}^{1} e^{-s^{2}\left(1-\sigma^{2}\right) / 4 \omega} d \sigma,
$$

where $a$ is a number.

In this manner, the class of analytic functions (11), depending on the parameter $a$, is assigned to $e^{\omega t^{2}}$. In the case of a non-positive real part of $\omega$, we can determine $\alpha$ in such a way that $x$ is the Laplace transform of $e^{\omega t^{2}}$. If the real part is positive, the Laplace transform does not exist. In that case another condition may be used to determine $\alpha$, e. g. that $x$ should be an odd function of $s$.

Rȩ̣u par la Rédaction le 12. 9. 1959

\section{The form of the solution of the Cauchy Problem over a group}

by

\section{R. E. EDWARDS (Reading)}

§ 0. Introduction and summary. Apart from certain extensions to be discussed briefly in $\S 6$, we shall for definiteness concentrate on what may be termed the "parabolic case" of the Cauchy Problem suggested by the heat (or diffusion) equation. The Cauchy Problem to be considered is therefore of the type

$$
D u_{t}=\dot{u}_{t} \quad(t>0)
$$

the dot indicating differentiation with respect to $t$, with the initial condition

$$
\lim _{t \rightarrow+0} u_{t}=f .
$$

Herein $u_{t}$ is, for each $t>0$, an element of some pre-assigned locally convex space $\varepsilon$ of real-valued functions on a group $X, D$ is a given endomorphism of $\varepsilon$, and the initial "data" $f$ is an element of a second pre-assigned locally convex space $\mathcal{D}$ of real-valued functions on $X$. We assume: (i) the existence and uniqueness of the solution; (ii) certain simple properties of the mapping $f \rightarrow u_{t}$; (iii) a few conditions of a very general nature concerning $D$ and $\varepsilon$; and (iv) the crucial condition that $D$ commutes with right translations. From this we deduce that the solution is necessarily of the form

$$
u_{t}=\mu_{t} * f
$$

each $\mu_{t}$ being a Radon measure on $X$. There are close connections between the results established below and those set forth by Hille ([4], p. 400-410), whose main aim is to exhibit the relations between solutions of the Cauchy Problem and the theory of semigroups. By comparison the present method is in some senses more general, uses fewer special assumptions, and accords to the convolution a more fundamental role.

The proof of the main theorem, which is given immediately after the hypotheses have been set forth at length in $\S 1$, occupies $\S 2$. The method is suggested by arguments used elsewhere (Edwards [2], [3]). 
In $\S 3$ certain consequences of the main theorem are detailed. Amongst these are some remarks about the apparent infinite velocity of transmission of effects governed by some equations of the type considered (the heat and diffusion equations, for example). It is shown that if one takes into account the finite sensitivity of measuring instruments, this objectionable feature is more apparent than real.

Calling to mind the connections with semigroup theory, we show in $\S 4$ that if $\mathcal{D}$, which in the main theorem is assumed to be suitably related to right-translations, is assumed further to be similarly related to left-translations, then the $\mu_{t}$ form a one-parameter semigroup with respect to convolution. This property is further exploited when $X$ is commutative by examining the Fourier transforms of the measures $\mu_{t}$; this occupies $\$ 5$.

Finally, in $\S 6$, we comment briefly on the possibility of extending the main arguments. The extensions contemplated include the wave equation

with initial conditions

$$
D u_{t}=\ddot{u}_{t}
$$

$$
\lim _{t \rightarrow+0} u_{t}=f, \quad \lim _{t \rightarrow+0} \dot{u}=f^{\prime} .
$$

The main illustrations are those in which $X$ is $R^{n}$ and $D$ is a suitable linear partial differential operator with constant coefficients, but the method of treatment shows no preference for these over the case in which $D$ is a convolution operator. If $D$ is allowed to be a distribution, the former case is included in the latter.

§ 1. Definitions and hypotheses. The group $X$ is assumed to be locally compact with left-invariant Haar measure $m$ and neutral element $e$ Following Bourbaki ([1a], p. 48) we denote by $\mathcal{X}\left(\right.$ resp. $\mathcal{K}_{+}$) the set of continuous real-valued (resp. positive real-valued) functions on $X$ having compact supports. For any function $f$ on $X$ and any $a \in X$ we define the left- (resp. right-) translate $L_{a} f$ (resp. $R_{a} f$ ) by $L_{a} f(x)=f(a x)$ (resp. $\left.R_{a} f(x)=f(x a)\right)$ for $x \in X$.

In respect of locally convex spaçes we again follow Bourbaki ([1a], p. 81) by saying that a locally convex space $\mathcal{F}$ satisfies condition (EC) if it is separated, and if in $F$ the closed convex envelope of a compact set is weakly compact. Note that this condition is fulfilled whenever $\mathcal{T}$ is separated and quasi-complete (the said envelope being then even compact). Regarding duality theory for locally convex spaces, we follow in general the notation and terminology of Bourbaki [1b].

In the sequel both $\mathcal{D}$ and $\varepsilon$ are assumed to be locally convex spaces of real-valued functions on $X$ such that the following conditions (A) and $(B)$ are to hold when therein we take $F$ to be either $D$ or $C$. It may be added parenthetically that the restriction $D \subset \mathcal{K}$ is one of convenience: if the solution of the Cauchy Problem extends naturally to wider classes of initial data, it usually does so in such a way that $u_{t}$ depends continuously upon $f$, and a knowledge of the dependence for $f$ belonging to $D$ suffices to determine completely the behaviour of its extension. Moreover, variants of the hypotheses are possible (see $\S 6$ ).

(A) F satisfies condition (EC) above, and the topology of 5 is finer than that of simple convergence on $X$.

(B) $\mathcal{T}$ is invariant under right-translations $R_{a}$, each $R_{a}$ induces a continuous endomorphism of $\mathcal{F}$, and for each $f \in \mathcal{F}$ the mapping $a \rightarrow R_{a} f$ is continuous from $X$ into $\mathcal{T}$.

Besides this, $D$ is to satisfy the further condition

(D) $D \subset \mathcal{X}$ and $D_{+}$(the set of positive functions in $(D)$ is uniformly dense in $\mathcal{K}_{+}$and contains an "approximate identity" $\left(g_{i}\right)$, i. e. a directed family such that

(D.1) $\quad G \equiv \operatorname{Sup}_{i} \int g_{i} d m<+\infty \quad(K \subset X, K$ compact $)$

and

(D.2)

weakly in $D$ for each $f \in \mathcal{D}$.

$$
\lim _{i}\left(g_{i} * f\right)=f
$$

The space $\mathcal{E}$ and the endomorphism $D$ are to be inter-related in the following way:

(E) $D$ is an endomorphism of $\mathcal{E}$ which commutes with all right-translations $R_{a}$.

The principal hypotheses concern the solution of the Cauchy Problem. These run as follows, $p$ denoting a pre-assigned positive function on $(0, \infty)$.

(CP) To each $f \in \mathcal{D}$ corresponds a unique solution of the Cauchy Problem

$$
\begin{gathered}
u_{t} \in \mathcal{E}, \quad D u_{t}=\dot{u}_{t} \quad(t>0), \\
\lim _{t->+0} u_{t}=f \text { simply on } X\left({ }^{1}\right), \\
p(t)^{-1} u_{t} \text { is bounded in } \varepsilon \text { for } t>0 .
\end{gathered}
$$

This solution has the following properties:

(CP.1) If $f \in D_{+}$, then $u_{t} \geqslant 0$ and $u_{t}(e) \leqslant \operatorname{Max} f$, whilst to each compact $K \subset X$ and each $t>0$ corresponds a number $c(K, t)$ such that

$$
\int K^{u_{t}} d m \leqslant c(K, t) \int f d m\left({ }^{2}\right) .
$$

(1) No harm is done by replacing (1.2) by the condition that $\lim _{t \rightarrow+0} u_{t}=f$ in any stronger sense commutating with right translations (possibly thereby making it easier to ensure uniqueness).

(2) The actual form of the function $e(K, t)$ is immaterial for the main arguments; see also $\S 3$. 

into $\varepsilon$.

(CP.2) For fixed $t>0$, the mapping $f \rightarrow u_{t}$ is continuous from $D$ In $\varepsilon$.

In connection with the growth condition (1.3), we note that it may be nullified by allowing $p(t) \equiv+\infty$ and interpreting $(+\infty)^{-1}$ as 0 . Uniqueness is known to require an effective growth condition for the case of the heat equation, and to be superfluous for the wave equation.

It is convenient to recall here that the convolution of two functions $g$ and $f$ is by definition

(1.4a) $\quad g_{*} f(x)=\int g(x y) f\left(y^{-1}\right) d m(y)=\int f\left(y^{-1} x\right) g(y) d m(y)$,

whilst the convolution $\mu^{*} f$ of a measure $\mu$ and a function $f$ is by definition

$$
\mu * f(x)=\int f\left(y^{-1} x\right) d \mu(y)
$$

in all cases which concern us, these integrals exist for all $x$.

Finally, we remind the reader. that the vague topology of measures is the weak topology resulting from their role in defining linear forms on $\mathcal{K}([1 \mathrm{a}]$, p. 60$)$.

§ 2. Statement and proof of the main theorem. We now prove

THEOREM 1. Suppose that $D$ and $\mathcal{E}$ satisfy (A) and (B), that $D$ satisfies also (D) and $\mathcal{E}$ also (E), and that (CP) is fulfilled. The conclusion is that to each $t>0$ corresponds a unique positive Radon measure $\mu_{t}$ on $X$ such that:

$$
\begin{gathered}
\mu_{t}(X) \leqslant 1 \quad(t>0) \\
\lim _{t \rightarrow+0} \mu_{t}(X)=1 \\
\lim _{t \rightarrow+0} \mu_{t}=\delta \text { vaguely; } \\
\lim _{t \rightarrow+0} \mu_{t}\left(U^{\prime}\right)=0
\end{gathered}
$$

for each neighbourhood $U$ of $e$ in $X, U^{\prime}$ being $X \cap C U$ :

$$
\begin{array}{ll}
\text { (M.5) } & u_{t}=\mu_{t^{*}} f \quad(f \in(\mathcal{D}, t>0), \\
\text { i.e. } &
\end{array}
$$

i. e.

$$
u_{t}(x)=\int f\left(y^{-1} x\right) d \mu_{t}(y)
$$

for $f \in \mathcal{D}, x \in X$ and $t>0$.

Remarks. (1) There is an analogous assertion in which right-translates are replaced by left-translates in the hypotheses: then in (M.5) we should have to replace $\mu_{t}^{*} f$ by $f_{*} \mu_{t}$, whilst in all the other conclusions $\mu_{t}$ should be replaced by the measure $\Delta \cdot \mu_{t}, \Delta$ being the modular function (Weil [7], p. 40) defined by

for general $k \epsilon \mathcal{X}$.

$$
\int k\left(x a^{-1}\right) d m(x)=\Delta(a) \int k(x) d m(x)
$$

(2) The uniqueness of $\mu_{t}$ follows immediately from (M.6), in view of (M.1) and (D).

(3) The uniqueness hypothesis in (CP) may demand the imposition of boundary conditions on the solutions. These may be absorbed into the definitions of $\mathcal{D}$ and $\varepsilon$, provided they are linear boundary conditions.

The proof of Theorem 1 will be based upon two lemmas.

LEMMA 1. Let $\mathcal{T}$ be either $D$ or $\varepsilon$, assumed to satisfy (A) and (B). If $f \in \mathcal{K}$ and $g \in \mathcal{F}$, then $g * f$ is identical with the abstract integral

$$
h=\int f\left(a^{-1}\right) \cdot R_{a} g \cdot d m(a),
$$

a weak integral with values in $\mathscr{T}$.

Proof. The weak integral exists because of (EC) (see [1a], p. 81, Proposition 2). By (A), the linear form $\delta_{x}: h \rightarrow h(x)$ (Dirac measure placed at $x$ ) is continuous on $\mathcal{F}$. Hence by definition of the weak integral, one has

$$
\begin{aligned}
h(x)=\left\langle h, \delta_{x}\right\rangle & =\int f\left(a^{-1}\right)\left\langle R_{a} g, \delta_{x}\right\rangle d m(a) \\
& =\int f\left(a^{-1}\right) \cdot R_{a} g(x) d m(a) \\
& =\int f\left(a^{-1}\right) g(x a) d m(a)=g * f(x) .
\end{aligned}
$$

Thus $h=g * f$, as was to be proved.

LEMma 2. Let $X$ be a locally compact space, $\left(\mu_{i}\right)$ a directed family of positive Radon measures on $X$ which satisfy

$$
\lim \sup _{i} \mu_{i}(X) \leqslant 1 \text {. }
$$

Suppose that $\left(\mu_{i}\right)$ converges vaguely to a measure $\mu$ such that $\mu(X)=1$. Let $A$ be the support of $\mu$. Then

$$
\begin{aligned}
& \lim _{i} \mu_{i}(X)=1, \\
& \lim _{i} \mu_{i}\left(U^{\prime}\right)=0
\end{aligned}
$$

for any open set $U \supset A$, where $. U^{\prime}=X \cap C U$.

Proof. Let $\varphi$ be any positive lower semicontinuous function on $X$. By definition ([1a], p. 104), if $\lambda$ is any positive Radon measure on $X$, $\int \varphi d \lambda$ is the supremum of $\int k d \lambda$ for $k \epsilon \mathcal{K}_{+}$minorizing $\varphi$. Thus $\lambda \rightarrow \int \varphi d \lambda$, being the upper envelope of continuous functions, is lower semicontinuous for the vague topology on the set of positive Radon measures. Consequently

$$
\int \varphi d \mu \leqslant \liminf _{i} \int \varphi d \mu_{i}
$$

whenever $\mu_{i} \rightarrow \mu$ vaguely (the $\mu_{i}$ being positive). If we now suppose that $\varphi \leqslant 1$ everywhere and $\varphi=1$ on $A,(2.4)$ and (2.1) combine to show that

$$
\lim _{i} \int \varphi d \mu_{i}=1 .
$$


Taking $\varphi=1$ everywhere, $(2.2)$ results. Taking $\varphi=\varphi_{U}$ (the characteristic function of $U$ ) and subtracting the result from $(2.2)$ we arrive at (2.3).

Proof of the theorem. Take a fixed $t>0$ and denote by $T_{t}$ the mapping of $\mathcal{D}$ into $\varepsilon$ which carries $f$ into $u_{t}$. By (CP.2), $T_{t}$ is continuous. Moreover the uniqueness part of (CP), combined with (B) and (E), entails that $T_{t}$ commutes with right-translations $R_{a}$; it is merely necessary to note that (1.3) remains true when $u_{t}$ is replaced by $R_{a} u_{t}$ (since $R_{n}$ is continuous and therefore bounded), that (1.2) is equivalent to

and that

$$
\lim _{t \rightarrow+0} T_{a} u_{t}=R_{a} f \text { simply on } X,
$$

$$
\begin{aligned}
\frac{d}{d t}\left(R_{a} u_{t}\right) & =\lim _{\varepsilon \rightarrow 0}\left(R_{a} u_{t+\varepsilon}-R_{a} u_{t}\right) / \varepsilon \\
& =\lim _{\varepsilon \rightarrow 0} R_{a} \cdot\left(u_{t+\varepsilon}-u_{t}\right) / \varepsilon \\
& =R_{a} \cdot \lim _{\varepsilon \rightarrow 0}\left(u_{t+\varepsilon}-u_{t}\right) / \varepsilon=R_{a} \dot{u}_{t},
\end{aligned}
$$

so that (1.1) remains undisturbed on replacing $u_{t}$ by $R_{a} u_{t}$.

By Lemma 1, with $\mathcal{F}=D$, we have

$$
g_{i} * f=\int f\left(a^{-1}\right) \cdot R_{a} g_{i} \cdot d m(a)
$$

for $f \epsilon \mathcal{D}$, and so, since $T_{i}$ is continuous,

$$
\begin{aligned}
T_{t}\left(g_{i} * f\right) & =\int f\left(a^{-1}\right) \cdot T_{t} R_{a} g_{i} \cdot d m(a) \\
& =\int f\left(a^{-1}\right) \cdot R_{a} T_{t} g_{i} \cdot d m(a) \\
& =\left(T_{t} g_{i}\right) * f=h_{i}^{*} f
\end{aligned}
$$

say, the last step being based upon an application of Lemma 1 with $\mathscr{F}=\mathcal{E}$. Using (D.1). and the continuity of $T_{t}$ once more, we derive

\section{(2.6)}

weakly in $\varepsilon$.

$$
T_{t} f=\lim _{i}\left(h_{i} f\right)
$$

Thanks to

$$
\operatorname{Sup}_{i} \int_{K} h_{i} d m \leqslant \operatorname{Sup}_{i} c(K, t) \int g_{i} d m<+\infty
$$

for each compact $K \subset X$. The measures $h_{i} m$ are thus vaguely bounded, so that the directed family $\left(h_{i} m\right)$ admits a vague limiting point $\mu_{t}$, which is necessarily a positive Radon measure on $X$. But then the directed family $\left(h_{i}^{*} f\right)$ admits $\mu_{t^{*}} f$ as a limiting point for the topology of simple convergence on $X$, as appears from $(1.4 \mathrm{~b})$. If $(\mathrm{A})$ is applied to $\varepsilon,(2.6)$ shows that (M.6) holds.
By (CP.1), if $f \epsilon \mathcal{D}_{+}$one has

$$
\int f\left(y^{-1}\right) d \mu_{t}(y)=u_{t}(e) \rightarrow f(e)
$$

as $t \rightarrow+0$, and

$$
0 \leqslant \int f\left(y^{-1}\right) d \mu_{t}(y)=u_{t}(e) \leqslant \operatorname{Max} f .
$$

Since $D_{+}$is uniformly dense in $\mathcal{K}_{+},(2.8)$ shows that $\mu_{t}(X) \leqslant 1$, which is (M.1), and (2.7) then leads to (M.3). Lemma 2 may now be applied, taking $A=\{e\}$ and $\mu=\delta$; (M.2) and (M.4) follow. This completes the proof.

§ 3. Remarks and consequences. (a) Condition (1.3) does of course entail that the measures $p(t)^{-1} \mu_{t}$ are vaguely bounded, i. e. that

$$
\operatorname{Sup}_{t>0} p(t)^{-1} \mu_{t}(K)<+\infty
$$

for each compact $K \subset X$. This is, however, much inferior to (M.1) in general. The estimate (3.1) may become valuable if in (CP.1) we were to weaken the inequality $u_{t}(e) \leqslant \operatorname{Max} f$ to

$$
u_{t}(e) \leqslant q(t) \cdot \operatorname{Max} f .
$$

In this case (M.1) would be replaced by $\mu_{t}(X) \leqslant q(t)$; (M.3), (M.5) and (M.6) would remain, but the proof of (M.2) and (M.4) would break down. On the other hand (D.1) could be shown to lead to

$$
\mu_{t}(K) \leqslant G c(K, t),
$$

and it would be a matter of inspection to decide which of (3.1) and (3.3) contains more information.

(b) For the heat equation in $R^{n}$, detailed results concerning the uniqueness of the solution and its representation in conformulation form are available. For a résumé and references, see Rosenbloom [6].

(c) It is not very easy to check the integral inequality in (CP.1) prior to knowing the form of the solution, and this even in the familiar special case of the heat equation over $R^{1}$. It is therefore interesting to note that the said inequality is often derivable as a consequence of the other hypotheses, at least whenever the solution can be said to exist uniquely in some weak sense for all initial conditions $f$ which are integrable and have compact supports. For such weak solutions, modifications of the sense of the initial conditions would be necessary, as also would the interpretation of $D$ (partial derivatives being interpreted in the sense of distributions, for example).

To illustrate the possibilities, we shall assume for simplicity that $D=\mathcal{X}$ and that $X$ is $\sigma$-compact. The main assumption will be that $T_{t}$ 
is extendable from $\mathcal{D}$ to the space $L_{c}^{1}$ of classes of integrable functions with compact supports; for $f$ in $L_{c}^{1}, T_{t} f$ is to be a class of locally integrable functions, and we assume that

(*)

$$
T_{t}(f * g)=\left(T_{t} f\right) * g
$$

for $g \in \mathcal{K}$ (or for suitably "dense" subsets of $\mathcal{K}$ ). This convolution equation cannot now be derived, as it was before, since we lack knowledge of suitably continuity properties of $T_{l}$.

Let $\cap 3$ be the Banach space of (classes of) integrable functions $f$ vanishing a. e. outside a fixed compact set $H \subset X ; 73$ is equipped with the norm induced by that of $L^{1}=L^{1}(m)$. In addition, let $\mathcal{L}$ be the space of (classes of) locally integrable functions $F$, equipped with the topology defined by the seminorms

$$
F \rightarrow \int_{K}|F| d m
$$

$K$ ranging over any countable base for the compact subsets of $X$. $\mathcal{L}$ is a Fréchet space. To establish the integral inequality in question, it suffices to show that the mapping $T_{t}$ is continuous from $\urcorner$ into $\mathcal{L}$, and for this it suffices to show that $T_{t}$ has a closed graph.

Suppose then that a sequence $\left(f_{n}\right)$ extracted from 73 converges to 0 , and that $\left(F_{n}\right)=\left(T_{t} f_{n}\right)$ converges in $\mathcal{L}$ to $F$. We need to show that $F=0$. Now, if $g \epsilon \mathcal{K}, f_{n} * g \epsilon \mathcal{K}$ and $f_{n} * g \rightarrow 0$ uniformly, and the first part of (CP.1) shows that $T_{t}\left(f_{n}{ }^{*} g\right) \rightarrow 0$ simply. By $(*)$, this reads $F_{n^{*}} g \rightarrow 0$ simply. But $F_{n} \rightarrow F$ in $\mathcal{L}$, and so $F_{n^{*}} g \rightarrow F^{*} g$ simply. Consequently $F^{*} g=0$ everywhere, and this for all $g \in \mathcal{K}$. This entails that $F$ is the zero class, as was to be proved.

Note finally that (*) will be satisfied if uniqueness of the solution of the Cauchy problem obtains in a suitable sense, the modified sense of the initial condition "commuting" with right-convolution by elements of $\mathcal{K}$, and if $D$ likewise commutes with right-convolution by elements of $\mathcal{X}$. The argument will be as in the proof of Theorem 1 , right-convolution replacing right-translation. (Right-convolution defines a continuous endomorphism of 2 ).

(d) If we know that the Cauchy Problem has a unique solution for (say) each bounded continuous $f$, and that (3.2) holds (the other hypotheses remaining as before), it is easily seen that (M.5) and (M.6) hold for all such $f$ by virtue of a simple continuity argument.

If further $D(1)=0$ (as in the case when $D$ is a partial differential operator), uniqueness guarantees that $f=1$ yields $u_{t}=1$, and then (M.6) gives $\mu_{t}(X)=q(t)$. In particular, in the original case $(q(t)=1)$, (M.1) and (M.2) may be simultaneously strengthened to

$$
\mu_{t}(x)=1 \quad(t>0) .
$$

(e) If $f \epsilon \mathcal{D}$, equations (M.1) and (M.6) yield

$$
\int\left|u_{t}(x)\right| d m(x) \leqslant \mu_{t}(X) \cdot \int|f(x)| d m(x),
$$

with equality if $f \geqslant 0$ :

$$
\int u_{t} d m=\mu_{t}(X) \cdot \int f d m
$$

(f) It often happens (as, for example, in the case of the heat equation over $X=R^{n}$ ) that if $f \geqslant 0, f \neq 0$, then $u_{t}(x)>0$ for all $x \in X$ and all $t>0$. This gives the impression that an infinite velocity of transmission is involved, a conclusion which would be physically objectionable. A closer look shows however that there is really no paradox, provided one makes allowance for the finite sensitivity of measuring instruments.

To see this we note that, after at most a change of scale, we can in the cases of physical interest arrange that $f \geqslant 0$. Suppose further that $f$ vanishes outside a set $A \subset X$ and is normalized by

$$
\int f d m=1 \text {. }
$$

The integral on the left may be thought of as a measure of the total initial heat content. If (3.4) holds, as it in fact does for the heat problem, $\left(3.5^{\prime}\right)$ expresses the conservation of total heat content. Take a neighbourhood $U$ of $e$ and a fraction $r(0<r<1)$ and consider the time lapse necessary before a fraction $r$ of the total heat is to be found outside of the neighbourhood $V=A U$ of $A$. A lower bound for $r$, in order that the said change may be observable, is set by the sensitivity of measurements, and until a certain minimal time has elapsed measurement cannot confirm a flow of heat out from its initial confinement in $A$ into the exterior of $V$. This condition requires that

$$
\int_{\nabla}, u_{t} d m \geqslant r \text {. }
$$

Now from (M.6) we find that the integral on the left of (3.7) is

$$
\int v^{r}, d m(x)=\int x A^{-1} f\left(y^{-1} x\right) d \mu_{t}(y) .
$$

If $x \in V^{\prime}$, then $x A^{-1} \subset U^{\prime}$, so that the left-hand side of (3.7) does not exceed

$$
\int_{\nabla^{\prime}} d m(x) \int_{U^{\prime}} f\left(y^{-1} x\right) d \mu_{t}(y)=\int_{U^{\prime}} d \mu_{t}(y) \int_{V^{\prime}} f\left(y^{-1} x\right) d m(x) \leqslant \mu_{t}\left(U^{\prime}\right)
$$

by virtue of (3.6) and left-invariance of $m$. Thus (3.7) entails

$$
\mu_{t}\left(U^{\prime}\right) \geqslant r .
$$

The relation (M.4) shows that (3.8), and a fortiori (3.7), can obtain only if $t \geqslant T(U, r)>0 . T(U, r)$ thus sets a lower limit to the time which 
must elapse before experiment can detect the flow of heat out of $A$ into the exterior of $V$.

As a simple illustration, take the one-dimensional heat equation

$$
\partial^{2} u / \partial x^{2}=\dot{u}
$$

over $X=R$. In this case $\mu_{t}$ has density (relative to $m$ ) given by

$$
\left.(4 \pi t)^{-1 / 2} \exp \left(-x^{2}\right) / 4 t\right) \text {. }
$$

If $U=(-a, a),(3.8)$ amounts to

$$
2 \pi^{-1 / 2} \int_{a_{/ 2} t^{1 / 2}}^{\infty} e^{-s^{2}} d s \geqslant r .
$$

This requires $t^{-1 / 2} a \leqslant C(r)$, where $C(r)>0$ depends only on $r$. Thus (3.8) entails $t \geqslant C(r)^{-2} a^{2}$. A time $T=C(r)^{-2} a^{2}$ must elapse before a measurable quantity (depending in amount on the sensitivity of measurements) will have flown outwards through a distance at least $a$ from its initial localization. This dependence of $T$ upon $a$ indicates an infinitely small speed, rather than an infinitely large one. It is also in agreement with the view of heat flow and diffusion as being subject to an underlying random motion of particles.

\$ 4. The semigroup property of the $\mu_{t}$. We supplement (B) applied to $\mathscr{D}$ by similar conditions pertaining to left-translations, and show that as a consequence the $\mu_{t}$ form a semigroup with respect to convolution. The supplementary condition is the following one.

$\left(\mathrm{B}^{\prime}\right) \mathcal{D}$ is quasi-complete and, for fixed $f \in \mathcal{D}$, the mapping $a \rightarrow L_{a} f$ is bounded and continuous from $X$ into $\mathcal{D}$.

The gain is recorded in

THEOREM 2. Suppose that the hypotheses of Theorem 1 are fulfilled, together with condition $\left(\mathrm{B}^{\prime}\right)$ above. Suppose also that for each $s>0$ one has

Then

$$
\operatorname{Sup}_{t>0} p(s+t) / p(t)<+\infty \text {. }
$$

$$
\mu_{s+t}=\mu_{s}^{*} \mu_{t} \quad(s>0, t>0) .
$$

Proof. Take a fixed $s>0$ and $f \in(D)$. Put $f^{*}=u_{s}=\mu_{s}^{*} f$. Thanks to $\left(\mathbf{B}^{\prime}\right.$ ) and (M.1) (applied with $t=s$ ), it is seen that $f^{*}$ again belongs to $D$ : the arguments are like those used in the proof of Lemma $1, f^{*}$ being the weak integral

$$
\int L_{a^{-1}} f \cdot d \mu_{s}(a)
$$

and $\left(\mathrm{B}^{\prime}\right)$ being adequate to ensure the existence of this integral as an element of $\mathcal{D}$.
We consider the solution of the Cauchy Problem with the initial state $f$ replaced by $f^{*}$. Because of uniqueness, it is easily verified that the resulting solution is none other than $t \rightarrow u_{s+t}(t>0),(4.1)$ ensuring that this candidate still saitsfies the growth condition (1.3). By Theorem 1 , therefore, $u_{s+t}=\mu_{t} * f^{*}$, i. e.

$$
\mu_{s+t} * f=\mu_{t} * \mu_{s} * f \text {. }
$$

This relation obtains for all $s>0, t>0$ and $f \epsilon \cap$. Condition (D) now forces upon us the conclusion (4.2).

§. The case in which $X$ is commutative. We assume here that the hypotheses of Theorem 2 hold, and that in addition $X$ is commutative, and proceed to exploit the relations (4.2) by taking the Fourier transforms of the measures $\mu_{t}$.

It will be convenient to define $\mu_{0}=\delta$ : then (M.1), (M.5), (M.6) and (4.2) hold for $s \geqslant 0, t \geqslant 0$. Further, (4.2) and (M.2)-(M.4) show that $t \rightarrow \mu_{t}$ is vaguely continuous for $t \geqslant 0$, together with certain consequences of this fact.

Introduce the group $Y$ dual to $X$ and denote by $(x, y)$ the character function. The Fourier transform of $\mu_{t}$ is the function on $Y$ defined by

$$
\hat{\mu}_{t}(y)=\int(x, y) d \mu_{t}(x) \text {. }
$$

It is clear from (M.1) that $\hat{\mu}_{t}$ is (for fixed $t \geqslant 0$ ) a continuous positive-definite function on $Y$, and that

$$
\left|\hat{\mu}_{t}(y)\right| \leqslant 1 \quad(t \geqslant 0, y \in Y) .
$$

Taking the Fourier transform of (4.2) we arrive at the equivalent relations

(5.3) $\quad \hat{\mu}_{s+t}(y)=\hat{\mu}_{s}(y) \cdot \hat{\mu}_{t}(y) \quad(s \geqslant 0, t \geqslant 0, y \in Y)$.

These relations determine completely the dependence of $\hat{\mu}_{t}(y)$ upon $t$ when $y$ is fixed, as the following theorem shows.

THEOREM 3. The hypotheses are those of Theorem 2, together with the commutative property of $X$. $Y$ being the group dual to $X$, there exists a continuous complex-valued function $Q$ on $Y$ such that

$$
\begin{gathered}
\operatorname{Re} Q(y) \geqslant 0 \quad(y \in Y), \\
\hat{\mu}_{t}(y)=\exp [-Q(y) t] \quad(y \in Y, t \geqslant 0) .
\end{gathered}
$$

Remarks. Before embarking on the proof of Theorem 3, it is interesting to compare it with the formally identical conclusion presented by Hille ([4], p. 405-406, especially formulae (20.8.7) and (20.8.8)). In the case considered by Hille, $X$ is $R^{n}$ and $D$ is a partial differential operator 
with constant coefficients. As a consequence a direct application of the Fourier transformation to (1.1) will lead to the required result. For, if $D$ is any convolution operator,

$$
D u=k * u,
$$

then formally (and justifiably if, for example, $k$ has a compact support and elements of $\varepsilon$ are suitably restricted in their rate of growth qua functions on $R^{n}$ ) we may transform (1.1) into

\section{with the solution}

$\left(5.5^{\prime}\right)$

$$
\hat{k} \cdot \dot{u}_{t}=\dot{\hat{u}}_{t}, \quad u_{0}=f,
$$

This corresponds formally to

$$
u_{t}=\mu_{t} * f, \quad \text { where } \hat{\mu}_{t}=\exp (t k) .
$$

Comparing with (5.5), we are led to identify $\hat{k}$ with $-Q$. A formal inversion of $\left(5.5^{\prime}\right)$ gives

$$
u_{t}(x)=\int_{x} \exp (-t Q(y)) \cdot \overline{(x, y)} \cdot \hat{f}(y) d y,
$$

which is equivalent to Hille's formula (20.8.7) already cited. Justification of these heuristic arguments presents no difficulty in the situation envisaged by Hille.

The following proof of (5.5) must proceed less directly since we do not wish to assume the legitimacy or sense of applying the Fourier transformation to (1.1) itself.

Proof of Theorem 3. This will call upon an auxiliary result, Lemma 3, which will be stated and proved after the main argument.

It is convenient to introduce the alternative notation $M_{y}(t)=\hat{\mu}_{t}(y)$, so that $M_{y}$ is a function on $I=[0,+\infty)$. From (5.3) and the relation $\mu_{0}=\delta$ we shall have

$$
\text { (5.6) } \quad\left|M_{y}(t)\right| \leqslant 1, \quad M_{y}(0)=1, \quad M_{y}(s+t)=M_{y}(s) M_{y}(t)
$$

for $s, t \in I, M y_{y}$ is thus a "character" of the additive semigroup $I$. The first and principal step is to show that $M_{y}$ is necessarily an exponential.

To do this, we begin by showing that $M_{y}$ is measurable. Since $\mu_{t}(X) \leqslant 1$ and $\mu_{t} \rightarrow \mu_{t_{0}}$ vaguely as $t \epsilon I$ converges to $t_{0} \epsilon I$, Lemma 3 shows that $\hat{\mu}_{t} \rightarrow \hat{\mu}_{t_{0}}$ weakly in $L^{\infty}(Y)$ in the same circumstances. Thus if $F \in L^{1}(Y)$, the function $\varphi_{F}$, defined by

$$
\varphi_{F}(t)=\int F^{\prime}\left(y^{\prime}\right) \mu_{t}\left(y^{\prime}\right) d y^{\prime}
$$

$\left(d y^{\prime}=\right.$ Haar measure on $\left.Y\right)$, is continuous. If we allow $F$ to run through a suitable sequence $\left(F_{n}\right)$ converging to the Dirac measure placed at a point $y \in Y$, continuity of $\hat{\mu}_{t}$ shows that $\varphi_{F_{n}} \rightarrow M_{y}$ simply on $I$, whence follows the measurability of $M_{y}$.

It is important to notice that

$$
\lim _{t \rightarrow+0} M_{y}(t)=1
$$

uniformly on compact sets of $y \in Y$. This is an easy consequence of (M.3) and (M.4). Combined with (5.6), it shows that $M_{y}$ is non-vanishing.

The measurability of $M_{y}$ and its non-vanishing property imply that in fact it is indefinitely differentiable on $I$ (right-hand derivatives at $t=0)$. The argument, which depends on the use of the Laplace transform, is probably well-known, but we include it for completeness. Thanks to (5.6) and measurability of $M_{y}$, the Laplace transform

$$
L_{y}(p)=\int_{0}^{\infty} M_{y}(t) e^{-p t} d t
$$

is defined and holomorphic for $\operatorname{Re} p>0$. Since $M_{y}$ is non-vanishing, $\boldsymbol{L}_{y}$ can vanish only for sparse sets of $p$; in particular we can certainly choose for any given $y$ a corresponding $p$ such that $L_{y}(p) \neq 0$. More than this is true, however; since

$$
L_{y}(p)=\int_{0}^{\infty} \hat{\mu}_{t}(y) e^{-p t} d t,
$$

continuity of $\hat{\mu}_{t}$ and uniform boundedness show (via Lebesgue's convergence theorem) that $L_{y}(p)$ is continuous in $y$ for any fixed real $p>0$. Hence, given $y_{0} \in Y$, there exists $p>0$ and a neighbourhood $W$ of $y_{0}$ such that

$$
L_{y}(p) \neq 0 \quad(y \in W) .
$$

This being so, use of the functional equation in (5.6), followed by an obvious change of variable, leads to

$$
M_{y}(t)=L_{y}(p)^{-1} e^{p t} \int_{0}^{\infty} M_{y}(s) e^{-p s} d s
$$

holding for $t \geqslant 0$ and $y \epsilon W, p$ being chosen so that (5.8) applies. From (5.9) it is easily seen step-by-step that $M_{y}$ is indefinitely differentiable on $I$.

Once the differentiability of $M_{y}$ is known, the functional equation in (5.6) gives for $M_{y}$ the simple differential equation

$$
M_{y}^{\prime}(t)=M_{y}^{\prime}(0) \cdot M_{y}(t)
$$


Bearing in mind the initial condition $M_{y}(0)=1$, we derive

$$
\hat{\mu}_{t}(y)=M_{y}(t)=\exp [-Q(y) t] .
$$

Boundedness of $M_{y}$, asserted in (5.6), shows that $\operatorname{Re} Q(y) \geqslant 0$.

It remains to show that $Q$ is continuous. Let $y_{0} \in Y$ and choose $W$ so that (5.8) obtains. It suffices to show that $Q \mid W$ is continuous. Now (5.10) and (5.9) give at once

$$
-Q(y)=M_{y}^{\prime}(0)=p-L_{y}(p)^{-1} .
$$

We have already seen that $L_{y}(p)$ is continuous in $y$ for any fixed real $p>0$, so that continuity of $Q \mid W$ is apparent.

Completion of the proof of Theorem 3 amounts now to establishing the auxiliary Lemma 3 .

LEMMA 3. Let $X$ be a locally compact commutative group with dual $Y$, and let $\left(\mu_{i}\right)$ be a norm-bounded directed family of Radon neasures on $X$, $\mu$ a bounded Radon measure on $X$. The following assertions are equivalent:

(a) $\mu_{i} \rightarrow \mu$ vaguely;

(b) $\mu_{i} \rightarrow \mu$ weakly (i.e. weakly in the dual of the space $C(X)$ of bounded continuous functions on $X$ which tend to zero at infinity);

(c) $\hat{\mu}_{i} \rightarrow \hat{\mu}$ weakly in $L^{\infty}(Y)$.

Proof. Let $H \in L^{1}(Y)$ and let

$$
h(x)=\int H(y) \cdot \overline{(x, y)} d y
$$

be its inverse Fourier transform. The generalized form of the Riemann-Lebesgue lemma affirms that $h \epsilon O(X)$. By the Lebesgue-Fubini-Tonelli theorem,

(5.11)

$$
\int h(-x) d \lambda(x)=\int H(y) \hat{\lambda}(y) d y
$$

for any bounded Radon measure $\lambda$ on $X$. Note that norm-boundedness of $\left(\mu_{i}\right)$ implies that of $\left(\hat{\mu}_{i}\right)$ relative to the customary norm on $L^{\infty}(Y)$

The implication (b) $\Rightarrow$ (a) is trivial, since $\kappa \subset C(X)$.

On the other hand, thanks to boundedness, (c) is equivalent to the requirement that

$$
\int H \hat{\mu}_{i} d y \rightarrow \int H \hat{\mu} d y
$$

for each $H$ belonging to a dense subset of $L^{1}(Y)$. It are known ([5] Section 37A, p. 147-148) that those $H \epsilon L^{1}(Y)$, for which $h \epsilon \mathcal{K}$, are dense in $L^{1}(Y)$. It therefore follows that (a) $\Rightarrow(\mathrm{c})$.

It remains to show that $(\mathrm{c}) \Rightarrow(\mathrm{b})$. But, thanks again to boundedness, (b) is equivalent to the demand that

$$
\int h(-x) d \mu_{i}(x) \rightarrow \int h \hat{\mu}(-x) d(x)
$$

for each $h$ belonging to a dense subset of $C(X)$. Such a set is formed by those $h \epsilon C(X)$ which belong also to $L^{1}(X)$ and for which $H=\hat{h}$ is in $L^{1}(Y)$. To verify this it suffices to first approximate any $h \in C(X)$ by $h^{\prime} \epsilon C(X) \frown L^{1}(X)$, and then to approximate $h^{\prime}$ by functions $h^{\prime \prime}=h^{\prime} * p$, where $p$ is continuous, positive definite and in $L^{1}(X)$. For any $h$ of this dense set, (5.11) shows that (c) implies (5.12). This completes the proof.

Remark. Lemma 3 generalizes a result due to Carathéodory for the ease in which $X$ is the circle group ([8], p. 82).

§ 6. Possible extensions. Arguments basically similar to those which precede apply to other problems of the same nature, the central argument being clearly that part of the proof of Theorem 1 which amounts to an appropriate representation theorem for continuous linear maps $T$ which commute with translations (right- or left-) in terms of convolution with a measure. As is clear from [3], this development is not necessarily tied down to the case in which $X$ is a full group. Moreover it is possible, and sometimes necessary, to drop the assumption, contained in (CP.1), that $T_{t}$ is positive. Instead one might assume that

$$
\begin{gathered}
\left|u_{t}(e)\right| \leqslant M_{i} \cdot \operatorname{Max}|f| \quad(f \in \mathcal{I}), \\
\int_{K}\left|u_{t}\right| d m \leqslant c(K, t) \cdot \int f d m \quad\left(f \in D_{+}\right) .
\end{gathered}
$$

The existence of the measure $\mu_{t}$ would then follow as before, as also would its boundedness; but it would no longer be true in general that $\mu_{t} \geqslant 0$, and (M.1) would take the weaker form

$$
\left|\mu_{t}\right|(X) \leqslant M_{t} \quad(t>0) .
$$

A further extension, which would in fact involve modifications of the type just contemplated, is illustrated by considering the Cauchy Problem for the "wave equation"

$$
D u_{t}=\ddot{u}_{t}
$$

with initial conditions

$$
\lim _{t \rightarrow+0} u_{t}=f, \quad \lim _{t \rightarrow+0} \dot{u}_{t}=f^{\prime} .
$$

For the semigroup aspects, see [4], p. 390-395.

To deal with this problem one would replace $D$ by a product $D \times D^{\prime}$ of two spaces, each similar to $\mathcal{D}$, and define $T_{t}: \mathcal{D} \times \mathcal{D}^{\prime} \rightarrow \mathcal{C}$ by

$$
T_{t}\left(f, f^{\prime}\right)=u_{t} \text {. }
$$

If we define $R_{a}$ on $\mathcal{D} \times \mathcal{D}^{\prime}$ by

$$
R_{a}\left(f, f^{\prime}\right)=\left(R_{a} f, R_{a} f^{\prime}\right),
$$


$T_{t}$ would commute with each $R_{a}$. However, $T_{t}$ would not be bilinear. This feature suggests falling back on an approach which is a priori very natural, and which reduces the problem to two partial problems each similar to that dealt with in $\$ 1-5$ above. It is merely necessary to decompose $u_{t}$ into a sum

$$
u_{t}=v_{t}+v_{t}^{\prime}
$$

where $v_{t}=T_{t}(f, 0)$ is the solution of (6.3) and (6.4) with $f^{\prime}=0$, and $v_{t}^{\prime}=T_{t}\left(0, f^{\prime}\right)$ is the solution of the same equations with $f=0$. The mappings

$$
\begin{aligned}
S_{t}: \mathcal{D} & \rightarrow \varepsilon, \quad S_{t} f=T_{t}(f, 0)=v_{t}, \\
S_{t}^{\prime}: \mathcal{D}^{\prime} \rightarrow \varepsilon, & S_{t}^{\prime} f^{\prime}=T_{t}\left(0, f^{\prime}\right)=v_{t}^{\prime}
\end{aligned}
$$

are each linear and commute with right-translations. Each may be treated by the methods of $\S \S 1-5$. The resulting representation theorem will be of the form

$$
u_{t}=\mu_{t} * f+\mu_{t}^{\prime} * f^{\prime},
$$

where $\mu_{t}$ and $\mu_{t}^{\prime}$ are bounded Radon measures. In general $\mu_{t}$ and $\mu_{t}^{\prime}$ will not be positive measures: this will depend partly on $X$ and partly on the boundary conditions (if any), and also of course on the nature of $D$.

\section{References}

[1a] N. Bourbaki, Intégration, Ch. I-IV, Paris 1952.

[1b] - Espaces vectoriels topologiques, Ch. III-V, Paris 1955.

[2] R. E. Edwards, On factor functions, Pacific Journ. Math. 5 (1955), p. $367-378$.

[3] - Representalion theorems for certain functional operators, Pasific Journ. Math. 7 (1957), p. 1333 - 1339 .

[4] E. Hille, Functional analysis and semigroups, American Math. Soc. Colloquium Pub. 31 (1948).

[5] L. Loomis, Abstract harmonic analysis, New York 1953.

[6] P. C. Rosonbloom, Linear equations of parabolic type with constant coefficients, Annals of Math. Study 33 (1954), p. 191-200.

[7] A. Weil, L'intégration dans les groupes topologiques et ses applications, Paris 1953.

[8] A. Zygmund, Trigonometrical series. Warsaw 1935.

$$
\text { Reçu par la Rédaction le 2. 10. } 1959
$$

\section{Projections in certain Banach spaces}

\author{
by \\ A. PELCZYNSKI (Warszawa)
}

It is a well known fact that every finitely dimensional subspace $Y$ of a $B$-space $X$ is complemented (1) in $X$. In general this property of finitely dimensional. subspaces does not remain valid for subspaces of an infinite dimension. The first example of a subspace $Y$ which has no complement in $C[0,1]$ was due to Banach and Mazur [2]. Further examples of non-complemented subspaces in $L_{p}, l_{p}(1 \leqslant p \neq 2), c_{0}, m, M$ and in other spaces were given by Murray [25], Sobczyk [30], Philips [29] and Komatuzaki [21], [22]. In many cases the fact that a subspace $Y$ is not complemented in a $B$-space $X$ depends only on the isomorphic properties of $X$ and $Y$. For example: no reflexive infinitely dimensional subspace of $C[0,1]$ has a complement (Grothendieck [16]); in an arbitrary $B$-space $X$ each subspace isomorphic to a space $C(S)$, where $S$ is a topological compact Hausdorff space extremally disconnected, has a complement (Nachbin [26], Goodner [15]).

Hence the following two problems arise naturally:

$1^{\circ}$ Given a $B$-space $X$, characterize the isomorphic types of complemented subspaces of $X$.

$2^{\circ}$ Given a $B$-space $X$, characterize the isomorphic types of such $B$-spaces $Z$ that every subspace of $Z$ isomorphic to $X$ is complemented in $Z$.

In section 2 of this paper we prove (Theorem 1) that every infinitely dimensional subspace complemented in $l_{p}(p \geqslant 1)$ or $c_{0}$ is isomorphic to $l_{p}$ or $c_{0}$ respectively. We do not know whether the space $m$ has the same property. Partial results in this direction are given in section 4 (Corollaries 7-9). In section 3 we consider the reciprocal problem. We prove that, if $Y$ is a subspace of $c_{0}$, or $l_{2}$, or $s$, or $m$, or $l_{p}(1 \leqslant p \neq 2)$ and $Y$ is isomorphic to $c_{0}, l_{2}, s, m$, or is isometrically isomorphic to $l_{p}$ $(1 \leqslant p \neq 2)$, then $Y$ has a complement.

(1) For the terminology and notation see section 1 . 